\title{
Correction to: LncRNA AFAP1-AS1 promotes tumorigenesis and epithelial- mesenchymal transition of osteosarcoma through RhoC/ROCK1/p38MAPK/Twist1 signaling pathway
}

\author{
Deyao Shi', Fashuai Wu', Shidai Mu², Binwu Hu¹, Binlong Zhong ${ }^{1}$, Feng Gao ${ }^{1}$, Xiangcheng Qing ${ }^{1}$, Jianxiang Liu', \\ Zhicai Zhang $^{1 *}$ and Zengwu Shao ${ }^{1 *}$
}

\section{Correction to: J Exp Clin Cancer Res https://doi.org/10.1186/s13046-019-1363-0}

In the original publication of this manuscript [1], Fig. $5 \mathrm{a}$ needs to be revised, and adjustments have also been made to the captions for Figs. 2, 4, 5 and S1 to improve clarity for the reader. The revised Fig. 5 and captions for Figs. 2,4 and $\mathrm{S} 1$ are shown below:

Fig. 2 Effect of AFAP1-AS1 knockdown on the apoptosis, cell cycle, migration, invasion, actin filament integrity and vasculogenic mimicry formation of OS cells. a and b AFAP1-AS1 knockdown induced apoptosis and resulted in G0/G1 cell cycle arrest. c and d AFAP1-AS1 knockdown inhibited migration and invasion ability of OS cells. e In the AFAP1-AS1 knockdown group, the expression of cleaved Caspase 3, Bax were increased and the expression of Bcl-2, Cyclin D1 and MMP-9 were decreased compared to the scrambled group. f AFAP1-AS1 knockdown inhibited the VM formation ability of OS cells. g AFAP1-AS1 knockdown in OS cells induced loss of actin filament integrity. The integrity and fluorescence

The original article can be found online at https://doi.org/10.1186/s13046019-1363-0.

* Correspondence: zhicaizhang@126.com; szwpro@163.com 'Department of Orthopaedics, Union Hospital, Tongji Medical College, Huazhong University of Science and Technology, 1277 Jiefang Road, Wuhan 430022, China

Full list of author information is available at the end of the article intensity of actin filament in osteosarcoma cells were obviously decreased. ${ }^{*} P<0.05$, ${ }^{* *} P<0.01$. In Fig. 2e / Fig. 4a / Fig. 4b / Fig. S1, the same GAPDH images were used to normalize in multiple panels under the same experimental treatments.

Fig. 4 Effect of AFAP1-AS1 knockdown on molecular expression of OS cells and AFAP1-AS1 knockdowninhibited EMT is mediated via RhoC/ROCK1/ p38MAPK/Twsit1 signaling pathway. a AFAP1-AS1 knockdown in OS cells led to significantly decreased expression of mesenchymal markers (Ncadherin and Vimentin) and increased expression of epithelial marker E-cadherin. b In the AFAP1-AS1 knockdown group, the expression of RhoC, ROCK1, p-p38MAPK and Twsit1 was decreased compared to the scrambled group. c RIP assay demonstrated that AFAP1-AS1 interacted with RhoC in OS cells. $d$, e and f Overexpression of RhoC in AFAP1-AS1 knockdown OS cells could rescue AFAP1AS1 downregulation-induced inhibition of cell migration, invasion, EMT, and the expression level of ROCK1, phosphorylated p38MAPK and Twist1 were rescued. $g$ and $h$ Both downregulating AFAP1-AS1 and RhoC, the expression of Twist and EMT of OS cells were inhibited further. ${ }^{*} P<0.05$, ${ }^{* *} P<0.01$, ${ }^{* * * *} P<0.001$. In Fig. 2e / Fig. 4a / Fig. 4b/ Fig. S1, Fig. 4f / Fig. 4d and Fig. $4 \mathrm{~g} /$ Fig. $4 \mathrm{~h}$, the same GAPDH images were used to 


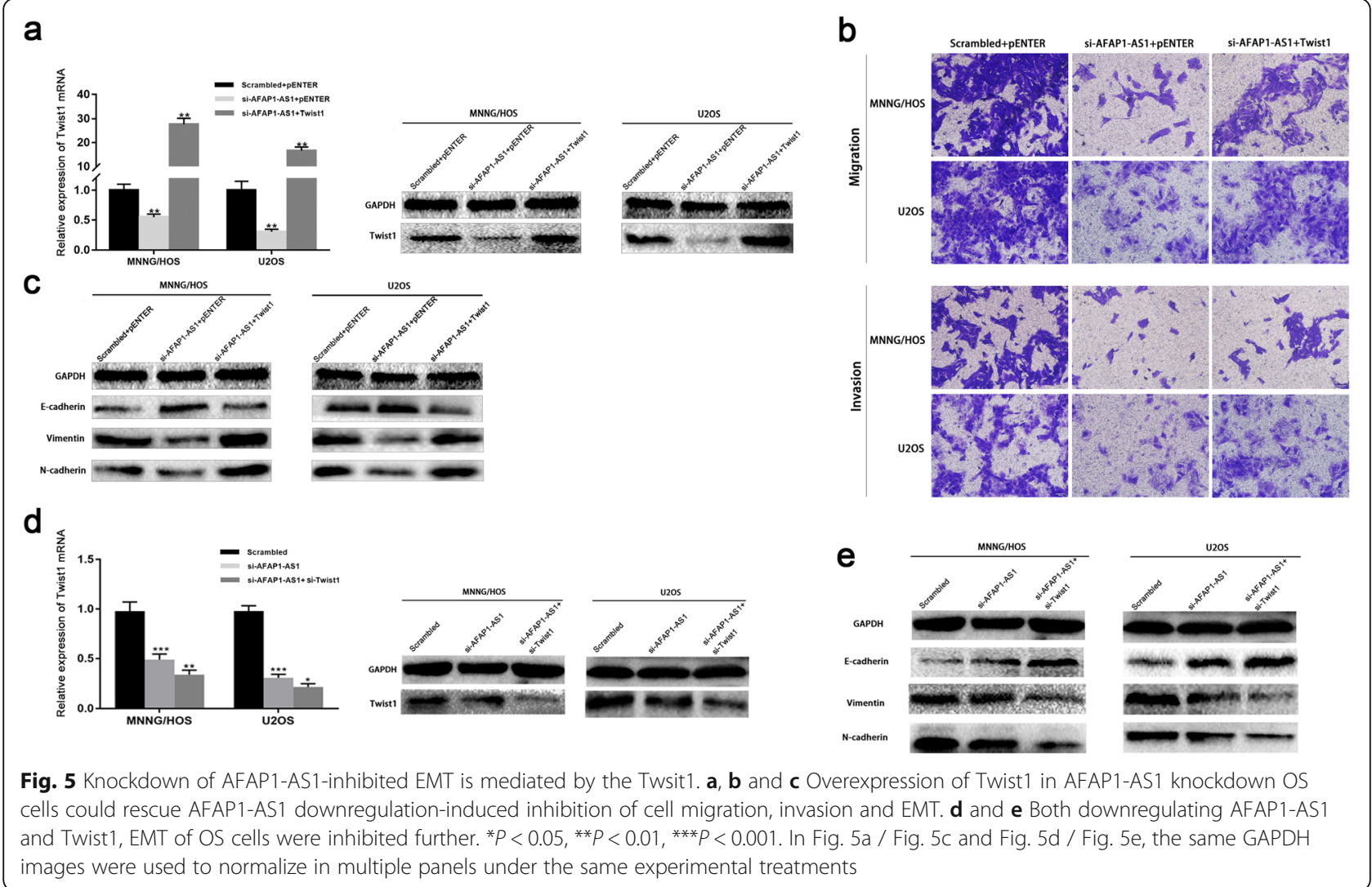

normalize in multiple panels under the same experimental treatments.

Figure S1. Knockdown of AFAP1-AS1 exerted no significant alteration on AFAP1 mRNA and protein expression. In Fig. 2e / Fig. 4a / Fig. 4b / Fig. S1, the same GAPDH images were used to normalize in multiple panels under the same experimental treatments.

\section{Author details}

'Department of Orthopaedics, Union Hospital, Tongji Medical College, Huazhong University of Science and Technology, 1277 Jiefang Road, Wuhan 430022, China. ${ }^{2}$ Institute of Hematology, Union Hospital, Tongji Medical College, Huazhong University of Science and Technology, 1277 Jiefang Road, Wuhan 430022, China.

Published online: 28 April 2020

\section{Reference}

1. Shi, et al. LncRNA AFAP1-AS1 promotes tumorigenesis and epithelialmesenchymal transition of osteosarcoma through RhoC/ROCK1/P38MAPK Twist1 signaling pathway. J Exp Clin Cancer Res. 2019;38:375. 